\title{
ANÁLISIS DEL USO DE TECNOLOGÍAS DE LA INFORMACIÓN Y COMUNICACIÓN EN EL SISTEMA EDUCATIVO
}

\section{ANALYSIS OF THE USE OF INFORMATION AND COMMUNICATION TECHNOLOGIES IN STUDENTS IN THE EDUCATIÓN SYSTEM}

\author{
Andrea Camila Torres Santiago* \\ ${ }^{1}$ Universidad Francisco de Paula Santander Ocaña, Colombia \\ https://orcid.org/0000-0001-5244-973X, E-mail: actorress@ufpso.edu.co
}

Resumen: El uso de las tecnologías de la información y comunicación son de gran importancia para el desarrollo de la humanidad, durante el siglo XX el impacto y el uso de esta se ha convertido en una prioridad en la práctica educativa y formación de educadores. La tecnología con fines educativos es una herramienta pedagógica en el que se combina la enseñanza por fuera del aula tradicional, buscando dinamismo e interacción para mejorar la asimilación del aprendizaje. El estudio cuantitativo permitió conocer el uso de las tecnologías implementadas por los estudiantes y se concluye que las mismas, son una nueva manera de adquirir aprendizaje mediante el uso de aulas virtuales, redes sociales, vídeos tutoriales, donde los educadores y padres de familias deberán conocer y asesorar a los estudiantes sobre el uso correcto de las tecnologías.

Palabras claves: Aprendizaje, Educadores, Estudiantes, Enseñanza, Tecnologías de la información y las comunicaciones.

\begin{abstract}
Information and communication technologies are of great importance for the development of humanity. During the 20th century, the impact and use of these technologies has become a priority in educational practice and the training of educators. Educational technology is a pedagogical tool that combines teaching outside the traditional classroom, seeking dynamism and interaction to improve the assimilation of learning. The quantitative study allowed to know the use of the technologies implemented by the students and it is concluded that the same ones, are a new way to acquire learning by means of the use of virtual classrooms, social networks, tutorial videos where educators and parents will have to know and to advise the students on the correct use of the technologies.
\end{abstract}

Keywords: Learning, Educators, Students, Teaching, Information and Communication Technologies 


\section{Introducción}

Las tecnologías de la información y la comunicación han avanzado de forma rápida con el paso del tiempo; actualmente es inevitable que un adulto o adolescente no use los teléfonos, computadoras, redes sociales, y conozcan su utilidad; estas se han convertido en un modelo de aprendizaje.

Los avances que ha tenido la tecnología con el transcurso del tiempo han sido buenos y muy significativos. Las tecnologías son un recurso que facilita la educación, es por ello por lo que está investigación se centra en el análisis del uso que los estudiantes les dan a las tecnologías de la información y comunicación, el uso de estas se hace fundamental para los educadores, abarcando distintos tipos de metodologías de enseñanza. Martín-Laborda (2005) plantea lo siguiente:

Internet ofrece cantidades inmensas de información que permiten enfocar los temas desde distintos puntos de vista. Las publicaciones electrónicas, las revistas digitales, las bases de datos y las bibliotecas virtuales que están disponibles en la Red, así como las listas de distribución (o de discusión) y los boletines de noticias, facilitan a los profesores la tarea de estar informados de los resultados de las investigaciones y proyectos. (p.10)

Por lo citado anteriormente, el uso de las tecnologías es fundamental para mantener informados a los profesores acerca de temas de gran importancia y en cómo le podría esto ayudar a los estudiantes, de manera que ellos lo usen para formarse y no para otras cosas, puesto que los estudiantes usan las tecnologías. pero no adecuadamente, los educadores deben investigar sobre la forma adecuada de que los estudiantes se informen y se formen usando las tecnologías.
El aprendizaje de los estudiantes no debe ser monótono, este debe ir cambiando según los avances que tenga la tecnología y el medio en el que nos encontramos. Hernández (2008) afirma que:" El aprendizaje de los estudiantes debe ser activo, deben participar en actividades en lugar de permanecer de manera pasiva observando lo que se les explica" (p.27). Fomentando el aprendizaje no solo dentro del aula de clases, sino también por fuera de está.

Así también, Echeburúa (2012) plantea que el abuso de las tecnologías de la información y comunicación es un riesgo, porque los jóvenes se convierten en adictos a ellas, y descuidan los estudios, así como también es un riesgo dejar desprotegido a un adolescente cuando está frente a estas tecnologías, puesto que pueden estar presentando ciber-acoso o accediendo a contenidos inapropiados.

Las redes sociales, como canal o fuente de información y comunicación, no son siempre buenas, la gran mayoría de las personas se encuentran desprotegidos, ya que la usan de manera inadecuada. Según Bacigalupe y Camara (2011)

La participación en redes sociales introduce una forma de transparencia que potencialmente pone, a su vez, en peligro la privacidad de esta adolescente. Además, la falta de control de los padres sobre las actividades online de la adolescente puede favorecer su desprotección, y verse víctima del ciber acoso. (p.11)

Las personas no son conscientes de los problemas que generan el mal uso de las redes sociales, así como el exponer fotografías inadecuadas, datos personales, la creación de cuentas ficticias, entre otros. Las instituciones deben brindar asesorías acerca del uso que le deben dar a las tecnologías de la información 
y comunicación, para que los estudiantes no se dejen llevar por lo que creen que está bien.

Para finalizar, el uso excesivo uso de las tecnologías produce una reducción en la comunicación cara a cara, puesto que los jóvenes se han convertido en adictos a las tecnologías donde prefieren entablar una conversación por una red social que entablarla personalmente; por tal razón se considera que se está haciendo un abuso de las tecnologías, Echeburúa (2012) nos plantea que el mal manejo de estás "Provoca aislamiento, induce ansiedad, afecta a la autoestima y le hace perder al sujeto su capacidad de control" (p.438). El aislamiento es provocado por las excesivas horas que un adolescente dedica a las redes sociales, perdiendo hábitos importantes para mejorar la calidad de vida: una buena conversación, practicar deportes, el leer un libro, etc.

Por tal razón, se expondrá el uso inadecuado que los estudiantes de la institución educativa Francisco Fernández de Contreras del municipio de Ocaña les dan a las tecnologías de la información y comunicación, y los problemas que trae esté para el aprendizaje.

\section{Marco Teórico}

Para lograr una mejor comprensión de cada una de las definiciones teóricas que rigen las tecnologías de la información y la comunicación en la educación y como se relacionan cada uno de ellos con los aspectos que en ella influyen. Por lo tanto, es necesario la creación de un contexto teórico en el que se pueda apoyar dicha investigación con soporte en las siguientes teorías.
En primer lugar, esta investigación abarca las siguientes teorías del aprendizaje, las cuales van enlazadas con el uso de las tecnologías de la información y

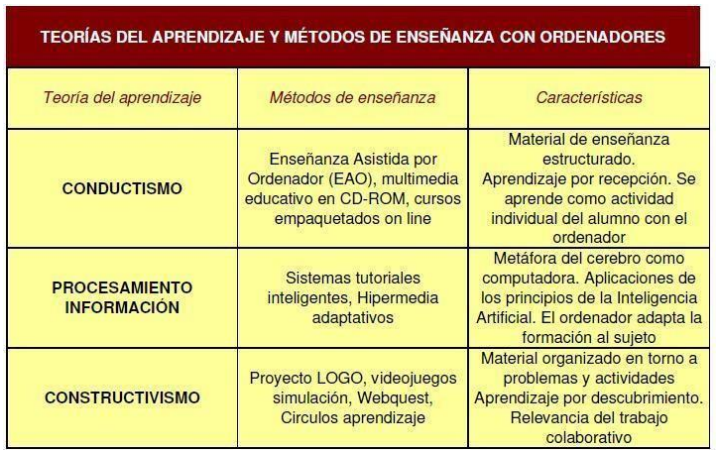

la comunicación.

Figura 1.Teorías del aprendizaje y métodos de enseñanza con ordenadores. Fuente: (Área Moreira, 2009).

Partiendo de que la teoría del aprendizaje se divide en 3 grandes pilares, los cuales se basan en la enseñanza con el uso de las tecnologías de la información y la comunicación. Estas tres teorías se basan en facilitar la adaptación de los estudiantes a la nueva era de las tecnologías y como usarla para el aprendizaje. La teoría del conductismo fundada por John Watson y propulsada por B.F. Skinner fue uno de los proyectos desarrollados para la tecnología educativa en los años cincuenta y sesenta, está teoría se caracterizaba por el surgimiento de las máquinas de aprendizaje programadas para la enseñanza; esta programación era la máxima expresión del diseño tecnológico aplicado a la acción formativa donde se fomentaba un material de trabajo más estructurado y la conducta del estudiante al realizar la actividad individual en un computador, así lo planteaba (Area Moreira, 2009). Dicha máquina o computador de enseñanza consistía según (Angulo, Vales, Acosta y García , 2015) en: 
Dicha máquina de enseñanza consistía en un dispositivo con una pantalla, en donde se presentaba secuencial y gradualmente a los estudiantes una serie de preguntas o de problemas acompañadas de cuatro posibles respuestas, entre las cuales el estudiante debía elegir la opción correcta, si lo hacía la máquina reproducía algún tipo de sonido como recompensa, en caso contrario, la máquina no avanzaba a la siguiente pregunta. (p.45-46).

Aunque, la teoría conductivista según (Luján Ferrer \& Salas Madriz, 2009) hizo importantes contribuciones en el inicio de la Guerra Fría, puesto que estos planteaban estrategias para el avance militar, la ciencia y la tecnología, se prestaba para dar un desarrollo educativo y militar entre los años 1940 y 1959. Aunque en la década de 1980 ese creo el enfoque conductista el cual trataba sobre la asociación de la tecnología con el análisis y modificaciones de la conducta.

Mientras que, la teoría constructivista según Hernández el cual cita a Jonassen (1991) dice que esta teoría "propone que el ambiente de aprendizaje debe sostener múltiples perspectivas o interpretaciones de realidad, construcción de conocimiento, actividades basadas en experiencias ricas en contexto" (p.27). Puesto que esta es una teoría que se basa en la construcción de conocimientos y de hacer auténticas cada una de las ideas que tengan los estudiantes.

\section{Metodología}

La investigación se llevó acabo como una investigación descriptiva, según (Sabino, 1992) esta radica en la descripción de algunas características fundamentales de conjuntos homogéneos de fenómenos, por lo que se enfocará en describir de manera analítica la estructura y el comportamiento de los estudiantes cuando usan las tecnologías de la información y la comunicación, esto con el fin de detallar la influencia y las características de las variables enunciadas en los objetivos el cual es fundamental para el desarrollo de esta investigación (p.47). Por tanto, esta investigación se realiza con enfoque cuantitativo, ya que se recolectaran datos y se hará un análisis estadísticos de las variables, con lo que se probará cada una de las hipótesis presentadas en el planteamiento del problema y los objetivos a cumplir en esta investigación; con este enfoque según (Fernández y Díaz, 2002) se determina la fuerza de asociación entre las variables de la investigación, la generalización y objetivación de los resultados a través de la muestra para hacer inferencia en toda la población en la que se desarrolló.

La población objeto de estudio son los estudiantes de décimo y once de la institución educativa Francisco Fernández de Contreras del municipio de Ocaña, Norte de Santander, los cuales están conformado por 113 estudiantes en décimo y 92 estudiantes en once. Como técnica se utilizó la encuesta, con la finalidad de recolectar información y como instrumento se realizó un cuestionario que consta de 9 preguntas. Una vez obtenida la información mediante la aplicación de las encuestas, se realizó un análisis estadístico a través de tablas y gráficas (cuantitativo) con las respuestas obtenidas.

\section{Resultados}

La educación en el siglo XXI se ha caracterizado por el avance del desarrollo tecnológico, el cual hace necesaria su incorporación en la educación, la tecnología debe orientarse para elevar la calidad del proceso de enseñanza- aprendizaje con las mismas y como integrarlas de manera tal que los planteles educativos trasciendan a lo tecnológico (Cabero, 2001). El plantel o institución objeto de estudio cuenta con 113 
estudiantes en el grado décimo y 92 en el grado once, estos estudiantes según el instrumento aplicado, el 93\% de ellos usan las tecnologías de la información y la comunicación, sean estas capacitadas por el docente o no, las usan con el fin de estar actualizados con temas internacionales, nacionales e institucionales, permitiendo de esta forma tener más información de distintos temas de interés, también porque el uso de estas tecnologías les permite una mejor comunicación con las demás personas, ya sean estos familiares, conocidos $\mathrm{o}$ desconocidos. Partiendo del referente teórico de las teorías de la enseñanza, se puede resaltar que el uso de las tecnologías en el siglo XXI es un requerimiento en los procesos académicos, ya que por medio de estar se facilita la obtención de conocimiento y mejor aprendizaje, en esta pregunta se puede notar la concepción de las tres teorías de las enseñanza que plantea (Area Moreira, 2009) las cuales son el conductismo que es la asociación de la tecnología con la educación y las modificaciones que trae la conducta con esto, también esta constructivismo que propone el ambiente de aprendizaje donde se sostiene múltiples perspectivas o interpretaciones de la realidad y finalmente el procesamiento de la información que es donde se compara la mente humana con un computador ya que se compara para la elaboración de modelos que expliquen la conducta humana.

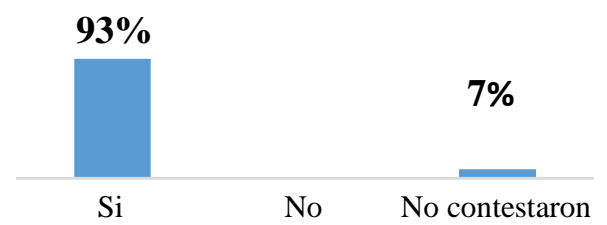

Figura 2. Uso de las tecnologías de la información y la comunicación. Fuente: Elaboración propia.
A continuación, se presenta información propia del instrumento aplicado con el que se reconocerán los objetivos y características claves para así analizarlas.

\subsection{Categorización sobre el uso de las TIC por los estudiantes.}

La propuesta EAO fundamentada por el referente teórico del conductismo, planteada por (Area Moreira, 2009) :"La EAO es una propuesta de individualización de la enseñanza que pretende a través del ordenador, que el alumno adquiera el conocimiento establecido de forma autónoma su propio ritmo de enseñanza “(p.57), seguidamente, es importante que los estudiantes adquieran un buen dominio de las tecnología, porque su uso es indispensable en la vida de los adolescente y más si estos son estudiantes. Las tecnologías brindan una manera fácil de adquirir conocimiento, adecuando como anteriormente se citaba un ritmo de enseñanza asertivo.

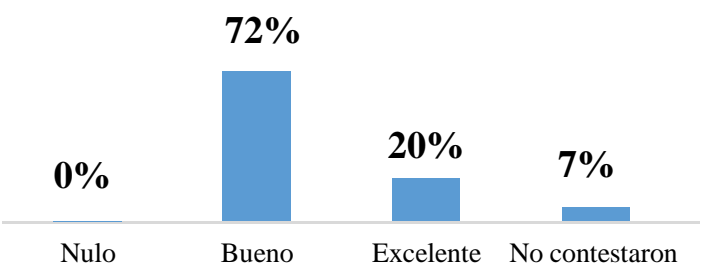

Figura 3. Dominio de las TIC de los estudiantes de décimo y once. Fuente: Elaboración propia.

El buen manejo de las tecnologías es relevante y se puede observar cuando el $20 \%$ de los estudiantes encuestados aluden que su manejo de las tecnologías es excelente y un $72 \%$ de ellos dicen que tienen un dominio bueno de estás. Por tanto, los estudiantes se deberán enfocar en relaciones como lo son formación mediante la información que nos brindan estás tecnologías. 
Los educadores también son parte fundamental de esta investigación, puesto que estos son los encargados de guiar a los estudiantes en los temas académicos y dar la mejor educación respecto el uso adecuado de las tecnologías para que los estudiantes no se vean afectados por ellos. Por tal razón, en el instrumento de investigación se cuestionaron a los estudiantes sobre la capacitación o conocimiento que tienen los educadores respecto a temas relacionados con las tecnologías.

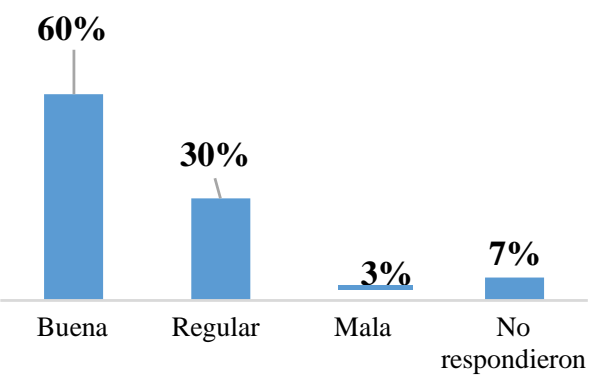

Figura 4. Conocimiento de los educadores respecto a Microsoft office. Fuente: Elaboración propia.

Estadísticamente, se observa que el $60 \%$ de los estudiantes consideran que el manejo de las herramientas ofimáticas es buena, ya que Microsoft office es esencial en la educación actualmente, ya que los trabajos deben ser presentados y guiados dependiendo una norma seleccionada por ellos, por otro lado hay $30 \%$ que consideran que tienen un conocimiento regular de las mismas, se podría decir que cuentan con las bases pero no la usan en su totalidad, también hay un 3\% de los estudiantes que desde su punto de vista consideran que los educadores tienen un mal manejo de Microsoft office.

\subsection{Determinación de los tipos de problemas que trae el uso constante de las TIC.}

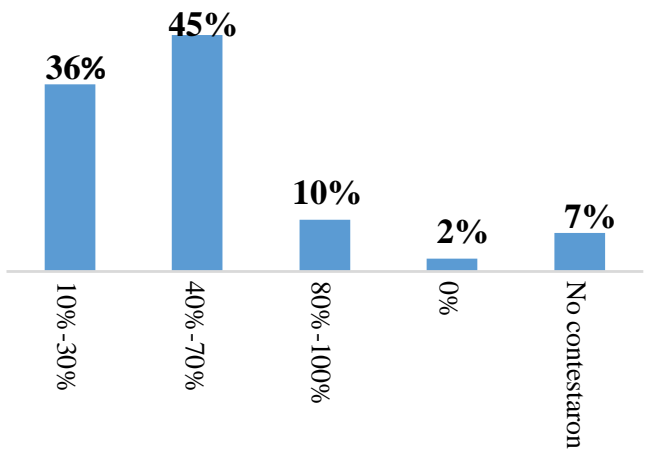

Figura 5. Porcentajes de la educación recibida en la institución acerca de las TIC según estudiantes de décimo y once. Fuente: Elaboración propia.

Los tipos de problemas que genera el uso de las tecnologías también se da por la poca educación brindada por parte de los directivos académicos en las instituciones, donde según el referente teórico constructivista es necesario que hayan herramientas donde sea opcional el uso del aula tradicional, generando un nuevo espacio donde tienen a su disposición herramientas tecnológicas, en la que los estudiantes logren afianzar o crear actividades innovadoras, obteniendo como resultado una construcción de conocimiento con el profesor como guía o mentor (Hernández, 2008), de acuerdo con lo anterior se obtiene que el $45 \%$ de los estudiantes consideran que la institución y los educadores brindan un porcentaje de educación acerca de las tic promedio, se puede decir que lo justo para una educación media, sin embargo, hay $36 \%$ de estos estudiante que consideran que no es suficiente donde su promedio se encuentra entre el $10 \%-30 \%$ de un $100 \%$, teniendo como base la teoría construccionista la institución no está colaborando para que los estudiantes sean capaces de construir nuevos 
conocimientos que le sirvan a ellos para su vida diaria.

\subsection{Afectaciones de las TIC en el rendimiento académico de los estudiantes.}

Según Hernández (2008) los estudiantes actualmente se deben enfrentar al constructivismo ya que esta ofrece un nuevo paradigma para esta nueva era de información. Con la llegada de las tecnologías de la información y comunicación los estudiantes no sólo tienen a su alcance el acceso a un mundo de información ilimitada de manera instantánea, sino que también tienen la posibilidad de controlar por ellos mismos la dirección de su propio aprendizaje, así como también tienen la libertad de experimentar nuevas cosas en línea.

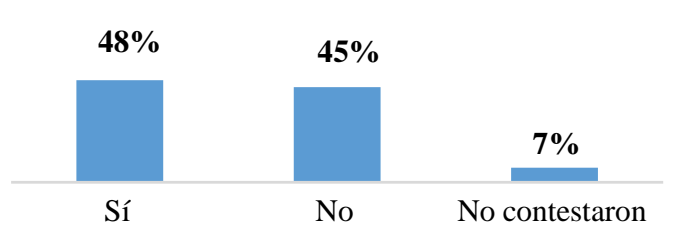

Figura 5. Afectación en el rendimiento académico por el uso constante de las tecnologías de la información y la comunicación. Fuente: Elaboración propia.

De acuerdo al instrumento aplicado en los estudiantes de décimo y once, el $48 \%$ considera que afectan las practicas académicas por el uso excesivo de las tecnologías, lo que quiere decir que la teoría de la enseñanza fundamentada por la teoría del constructivismo no se ha implementado de buena manera en los estudiantes, aunque la diferencia con los estudiantes que respondieron que no los afectaba el uso de las tecnologías es del 3\%, por lo tanto, hay que brindar apoyo o hacer más conciencia sobre el uso de las tecnologías.

\section{Conclusiones}

Las tecnologías de la información y la comunicación son de gran ayuda para la formación de los estudiantes en la educación, donde abarcan el uso de medio electrónicos para facilitar el acceso a información y de esta manera complementar el aprendizaje que se brinda en las aulas tradicionales; teniendo en cuenta que el uso de estás puede generar afectaciones en el rendimiento académico, pues no se asesoran a los estudiantes sobre el buen dominio y el uso de ellas, que en su efecto pueden generar problemas $\mathrm{u}$ afectaciones en la vida diaria, por lo tanto en todos los planteles educativos se debe ampliar programas de capacitación sobre la educación en el manejo de las tecnologías a todo el estudiantado.

Los educadores como responsables del aprendizaje y formación de los estudiantes deben ser capaces de manipular los recursos tecnológicos en los procesos de enseñanza, para así poder capacitar a los estudiantes sobre el uso de las tecnologías, enfocándose en el desarrollo de los programas que eviten la monotonía y fomenten el interés por la investigación, así se informan y se forman los estudiantes.

\section{Referencias}

Area Moreira, M. (2009). Introducción a la tecnología educativa. España. Recuperado el 08 de Octubre de 2018, de https://www.researchgate.net/publication /267362779_Introduccion_a_la_Tecnolo gia_Educativa 
Bacigalupe, G., \& Camara, M. (2011). Adolescentes digitales; el rol transformador de las redes. Bizkailab, 1112. Recuperado el 14 de Septiembre de 2018 , de https://www.bizkailab.deusto.es/wpcontent/uploads/2012/04/5749-Report01-art\%C3\%83\%C2\%ADculoAdolescentes-Digtales.pdf

Cabero, J. (2001). Tecnología educativa (Vol. 2). Madrid, España: Síntesis.SA. Recuperado el 30 de Noviembre de 2018, de

https://s3.amazonaws.com/academia.edu .documents/39137939/9_resena_2Julio_ Cabero.pdf?AWSAccessKeyId=AKIAI WOWYYGZ2Y53UL3A\&Expires $=154$ 3606613\&Signature $=$ QwyQBsmna84LX eX4bgW1713aRp4\%3D\&responsecontentdisposition=inline $\% 3 \mathrm{~B} \% 20$ filename $\% 3$ D9_resena_2Julio_Cabero.

Echeburúa, E. (10 de Diciembre de 2012). Factores de riesgo y factores de protección en la adicción a las nuevas tecnologías y redes sociales en jóvenes y adolescentes. Revista Española de Drogodependientes, 435-447. Recuperado el 16 de Septiembre de 2018, de

https://www.aesed.com/descargas/revist as/v37n4_5.pdf

Fernández, P., \& Díaz, P. (2002). Investigación cuantitativa y cualitativa. 76-78. Recuperado el 20 de Octubre de 2018, de https://www.fisterra.com/gestor/upload/ guias/cuanti_cuali2.pdf

González, H. (24 de Junio de 2015). El buen uso de la tecnología. Diario Occidente. Recuperado el 5 de Noviembre de 2018, de https://occidente.co/el-buen-uso-dela-tecnologia/
Hernández Requena, S. (2 de Octubre de 2008). El modelo constructivista con las nuevas tecnologías: aplicado en el proceso de aprendizaje. RUSC. Revista de universidad y sociedad del conocimiento, 5,26-35. Recuperado el 18 de Septiembre de 2018, de http://www.redalyc.org/articulo.oa?id=7 8011201008

Hernández Requena, S. (2 de Octubre de 2008). El modelo constructivista con las nuevas tecnologías; aplicado en el proceso de aprendizaje. RUSC.Revista de universidad y sociedad del conocimiento, 5, 26-35. Recuperado el 18 de Septiembre de 2018, de http://www.redalyc.org/articulo.oa?id=7 8011201008

Martínez Toribio, J. M. (30 de Enero de 2014). Uso adecuado de la tecnología. UNIVERSIDAD NACIONAL DE INGENIERÍAS. Recuperado el 5 de Noviembre de 2018, de https://www.uni.edu.ni/Articulo/Ver/Uso -adecuado-de-la-tecnologia

Martín-Laborda, R. (2005). Las nuevas tecnologías en la educación. AUNA, 10. Recuperado el 18 de Septiembre de 2018, de

http://giovannipf.260mb.net/tecnologiae nlaeducacion.pdf?i=1

Sabino, C. (1992). El proceso de investigación. Caracas, Venezuela: Panapo. Recuperado el 20 de Octubre de 2018, de https://metodoinvestigacion.files.wordpr ess.com/2008/02/el-proceso-deinvestigacion_carlos-sabino.pdf 